\title{
Atypical case of the postpartum posterior reversible encephalopathy associated with acute pulmonary edema
}

\author{
Hicham Bakkali ${ }^{1,}$, , Salahedine Massou ${ }^{1}$, Elhassani Mehdi ${ }^{2}$, Abdellah Babahabib ${ }^{2}$, \\ Khalil Aboulaala ${ }^{1}$, Hicham Balkhi ${ }^{1}$, Jaouad Kouach ${ }^{2}$ \\ ${ }^{1}$ Department of Anesthesiology and Critical Care, Military Training Hospital Med V, Rabat, Morocco \\ ${ }^{2}$ Department of Gynecology-obstetrics, Military Training Hospital Med V, Rabat, Morocco
}

\section{Email address:}

hbakkali@ymail.com (H. Bakkali), salah_masson80@hotmail.com (S. Massou), babahabib2003@hotmail.com (A. Babahabib), khalil_akram98@yahoo.fr (K. Aboulaala),balkhih@hotmail.com (H. Balkhi),kouach_jaouad@yahoo.fr (J. Kouach)

\section{To cite this article:}

Hicham Bakkali, Salahedine Massou, Elhassani Mehdi, Abdellah Babahabib, Khalil Aboulaala, Hicham Balkhi, Jaouad Kouach. Atypical Case of the Postpartum Posterior Reversible Encephalopathy Associated with Acute Pulmonary Edema. Science Journal of Clinical Medicine. Vol. 3, No. 1, 2014, pp. 1-3. doi: 10.11648/j.sjcm.20140301.11

\begin{abstract}
The Posterior Reversible Encephalopathy Syndrome (PRES) is a clinical and radiological entity involving varying degrees of neurological symptoms to neurological abnormalities in parietal-occipital white matter. We report a case of reversible encephalopathy of late posterior postpartum encephalopathy as an association with acute pulmonary edema, in a young woman without any significant medical history. The physiopathology, diagnosis and treatment were discussed.
\end{abstract}

Keywords: Posterior Reversible Encephalopathy, Postpartum, Tocolysis, Nicardipine, Acute Pulmonary Edema

\section{Introduction}

The PRES is a clinical and radiological entity associating varying degrees, headaches, impaired consciousness, seizures and visual disturbances to neurological and radiological abnormalities of the parietal-occipital white matter [1,3]. Its association with an acute pulmonary edema (APE) has never been established. It is a reversible syndrome, unless if there is an early taking over.

\section{Observation}

A woman aged 23 years, primiparous, without any pathological history, except a premature childbirth threat, 34 weeks due to amenorrhea (AW). This woman was treated efficiently by one tocolysis with nicarpidine and cesarean delivery under spinal anesthesia at $38 \mathrm{AW}$ for a commitment default to full dilatation of a new born of masculine sex. On the seven days, after the postpartum, the patient was admitted at emergency with manifestations of headaches, dizziness and dyspnea. Once admitted, she presented general seizures. She was treated immediately by mizadolam, a dose of phenobarbital $10 \mathrm{mg} / \mathrm{kg}$ and sodium valproate $1,5 \mathrm{~g} /$ day.

After a formal requirement, the clinical examination revealed an afebrile and obsessed patient with Glasgow coma score at 13 without any neurological deficit. This patient has tachycardia in $130 / \mathrm{min}$, with a hypertensive peak at $180 / 110 \mathrm{mmHg}$, respiratory rate at 40 cycles/min and one SPO2 of $88 \%$ under a high concentration. Pulmonary examination revealed diffuse crackles in both lung fields with frothy secretions and whitish. Initial investigation showed pulmonary edema with radiograph in butterfly wings on chest radiograph. ECG and troponine rate did not present abnormalities. Emergency treatment consisted of non-invasive ventilation with positive end-respiratory pressure and administration of injectable furosemide with a total dose of $80 \mathrm{mg}$ as well as a loading dose of magnesium sulfate $(1 \mathrm{~g} / 30 \mathrm{~min})$; then after $1 \mathrm{~g} / \mathrm{h}$ till the disappearance of diffuse crackles with improvement in respiratory level as well as hemodynamic and neurologic one, and finally an assessment was made. The elecroencephalography (EEG) showed diffused periodic activity within a short period (figure 1).

Brain computed tomography, lumbar puncture and laboratory tests were unremarkable apart from a rate of brain natriuretic peptide (BNP) of $457 \mathrm{pg} / \mathrm{ml}$, confirming the hemodynamic nature of APE. Angio MRI was in favor of a PRES postpartum (figure 2).

A treatment with anti-platelet and sodium valproate 
$1,5 \mathrm{~g} /$ day, was maintained. The evolution was marked by the total disappearance of symptoms. After 10 days, Control EEG was normal.

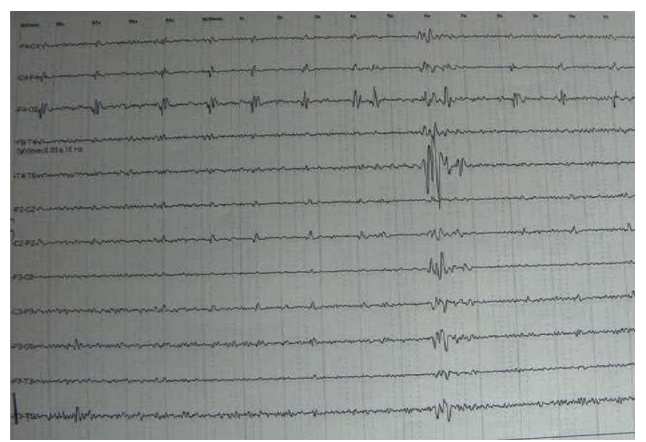

Figure 1. Electroencephalography showing Periodic short-interval diffuse paroxysmal patterns.

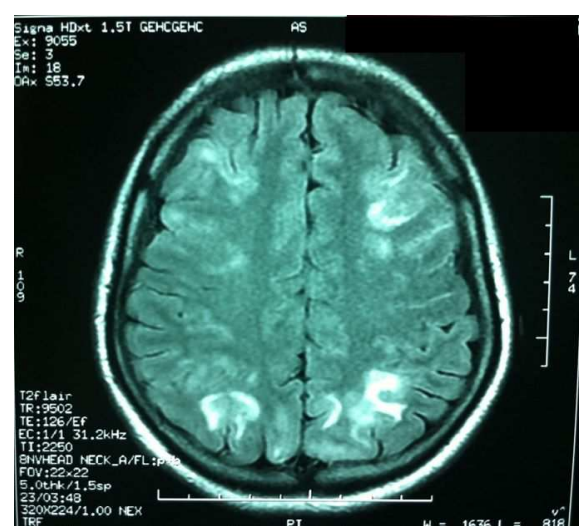

(a) (b) (c)

Figure 2. Brain MRI, T2 fluid-attenuated inversion recovery, showing bilateral assymetrique frontal parieto-occipital hypersignal.

\section{Discussion}

PRES replaced various names i.e.: hypertensive encephalopathy, posterior regressive leuko-encephalopathy or parietal-occipital encephalopathy [1]. It refers to a clinical and radiological syndrome partnering with varying degrees, four cardinal symptoms: headaches, impaired consciousness, and seizures, visual disturbances ranging from visual field to cortical blindness and abnormal white matter mainly parietal-occipital.

The PRES refers to young patients, most often young females, with sometimes serious comorbidity [2]. Pregnancy and per partum are conditions encouraging occurrence of such a syndrome in our patient [1]. On the pathologic level, we suggest one dysfunction of the blood brain barrier by passing self-capacity of cerebral pressure perfusion or endothelial cytotoxicity, responsible for the alteration of the blood brain barrier (BBB) [3]. These two mechanisms are held responsible for a vasogenic edema in the white matter, involving the clinical and radiological manifestations of PRES [1]. In the case of per partum, there are other additional mechanisms, especially capillary hyperpermeability of pregnancy, blood pressure and the role of substances modulating the vascular tone such as: hypersensitiveness to endogenous vasopressor agents, the reduction of prostaglandins and cytotoxic factor of placental origin, responsible for endothelial cell dysfunction [1, 3, 4]. On the topographic plan, the prevalence of disease is explained by the developing less important at the level of the sympathetic system that is neurogenic response intending to compensate for impaired endothelial myogenic lesions. The result is a hyper perfusion which, associated with the alteration of BBB and hyper arterial pressure, leads to a vasogenic edema. Etiologies associated to PRES are multiple, dominated by exposures to the toxic agents and acute hypertensive crisis. History of our patient is the concept of calcium channelblockers tocolytic taking (nicardipine) whose effect is known as setting hypertensive crisis [5].

Some authors report cases of APE in parturient that underwent tocolysis by nicarpidine $[6,7]$. The use in epidural of local anesthetics derived from cocaine which safety cannot be eliminated could be implicated in the occurrence of this syndrome in our case [1, 3, 4].The typical clinical manifestations of PRES associate impaired consciousness, seizures, headaches, visual disturbances, nausea, vomiting and focal neurological deficits [1]. The hypertension is present in the initial phase of taking over in 67 to $80 \%$ of the patients $[1,8]$. It is every outside hypertensive etiology that is considered a cause of PRES. In our patient, in addition to the neurological symptoms and Arterial hyperpression (AHP), one APE has complicated the clinical picture. The high rate of BNP and the improvement under diuretics made us to confirm the hemodynamic origin. The mechanism of APE can be assimilated to the one of PRES by the alteration of alveolar capillary membrane or simply the hypertensive crisis.

MRI is a key element of diagnosis [3, 4, 5, 8, 9]. It helps to rule out other possibilities of diagnosis i.e. myocardial venous and arterial cerebral thrombophlebitis as well as brain encephalitis. Indeed, it expresses abnormalities of parietal occipital, frontal, cerebral cortex and even in cortical signal, hypo intensive in T1, hyper intensive in $\mathrm{T} 2$ and FLAIR. The fast regression of the lesions showed a vasogenic edematous character of the lesions confirming the diagnosis. The evolution is usually favorable with total regression of symptomatology after some days, under condition of early diagnosis and appropriate treatment (antihypertensive treatment, magnesium sulfate, stop of suspect's toxicity treatment).

\section{Conclusion}

PRES of postpartum is still largely unknown, although it is relatively common, the evolution is good in short and long term unless if there is an early taking over.

PRES-APE association has never been established. The possibility of local anesthetics and nicardipine in per partum are the particularity of this observation. 


\section{Acknowledgments}

Our sincere acknowledgments are due to Mrs Saidi Elisabeth for the translation of this present article.

\section{References}

[1] Etesse. B, Letouzey. V, Roger. C, Lefauconnier. A, Ripart. J. Epidural analgesia is not the only cause of peripartum central neurologic symptoms. Report of one case of posterior reversible encephalopathy syndrome Ann Fr Anesth Reanim 2011;30:57-60

[2] Lee VH, Wijdicks EF, Manno EM, Rabinstein AA: Clinical spectrum of reversible posterior leukoencephalopathy syndrome. Arch Neurol 2008; 65:205-210.

[3] Legriel S, Pico F, Bruneel F, Troché. G, Bedos JP. Des pathologies encéphaliques à connaître: Syndrome d'encéphalopathie postérieure réversible. Reanimation 2011; 20:368- 378 .

[4] Onrubia X, Lluch-Oltra A, Armero R, Higueras R, Sifre C, Barbera M. Posterior reversible encephalopathy syndrome after caesarean delivery. Anesth Analg 2007;104:746-7.
[5] Adnet P, Krivosic-Horber R. Calcium inhibitors and anesthesia. AnnFr Anesth Reanim 1988;7:494-505.

[6] Philippe HJ, Le Trong A, Pigeau H, Demeure D, Desjars P, Esbelin J, Caroit Y, Winer N. Acute pulmonary edema occurred during tocolytic treatment using nicardipine in a twin pregnancy. Report of three cases. J Gynecol Obstet Biol reprod $2009 ; 38: 89-93$.

[7] Janower S, Carbonne B, Lejeune V, Apfelbaum D, Boccara F, Cohen A. Acute pulmonary edema during preterm labor: role of nicardipine tocolysis (three cases). J Gynecol Obstet Biol Reprod. 2005;34(8):807-12.

[8] Wernet A, Benayoun L, Yver C, Bruno O, Mantz J. Isolated severe neurologic disorders in post-partum: posterior reversible encephalopathy syndrome. Ann Fr Anesth Reanim 2007; 26:670-3.

[9] Yoon SD, Cho BM, Oh SM, Park SH, Jang IB, Lee JY. Clinical and radiological spectrum of posterior reversible encephalopathy syndrome. J Cerebrovasc Endovasc Neurosurg. 2013 Sep;15(3):206-13. 